\title{
Entry, Descent, and Landing Operations Analysis for the Genesis Entry Capsule
}

\author{
Prasun N. Desai* \\ NASA Langley Research Center, Hampton, VA, 23681-2199 \\ Daniel T. Lyons ${ }^{\dagger}$ \\ Jet Propulsion Laboratory, Pasadena, CA, 91109-8099
}

\begin{abstract}
On September 8, 2004, the Genesis spacecraft returned to Earth after spending 29 months about the sun-Earth libration point $\left(L_{1}\right)$ collecting solar wind particles. Four hours prior to Earth arrival, the sample return capsule containing the samples was released for entry and subsequent landing at the Utah Test and Training Range. This paper provides an overview of the entry, descent, and landing trajectory analysis that was performed during the mission operations phase leading up to final approach to Earth. The final orbit determination solution produced an inertial entry flight-path angle of $\mathbf{- 8 . 0 0 2} \mathbf{~ d e g}$ (which was the desired nominal value) with a 3- $\sigma$ error of $\pm 0.0274 \mathrm{deg}$ (a third of the requirement). The operations effort accurately delivered the entry capsule to the desired landing site. The final landing location was $8.3 \mathrm{~km}$ from the target, and was well within the allowable landing area. Overall, the Earth approach operation procedures worked well and there were no issues (logistically or performance based) that arose. As a result, the process of targeting a capsule from deep space and accurately landing it on Earth was successfully demonstrated.
\end{abstract}

\section{Introduction}

G ENESIS, the fifth of NASA's Discovery class missions, was launched on August 8, 2001. It was the first mission to return samples from beyond the Earth-moon system. Genesis was inserted into a halo orbit about the sunEarth libration point $\left(\mathrm{L}_{1}\right)$, where it collected solar wind particles over a period of approximately 29 months. The solar wind particles were collected in collector arrays, which were exposed by opening the capsule (Fig. 1). The arrays faced the sun, and the particles were trapped in a silica-based material. The collector arrays were retracted at the end of the collection period, and the capsule was closed. Reference 1 gives an overview of the Earth return trajectory strategy.

\footnotetext{
*Senior Engineer, Systems Engineering Directorate, 1 N. Dryden St., MS 489, prasun.n.desai@nasa.gov, Associate Fellow AIAA.

†Senior Aerospace Engineer, Inner Planet Dynamics Section, 4800 Oak Grove Dr., M/S 301-121, daniel.t.lyons@jpl.nasa.gov, AIAA Member.
} 


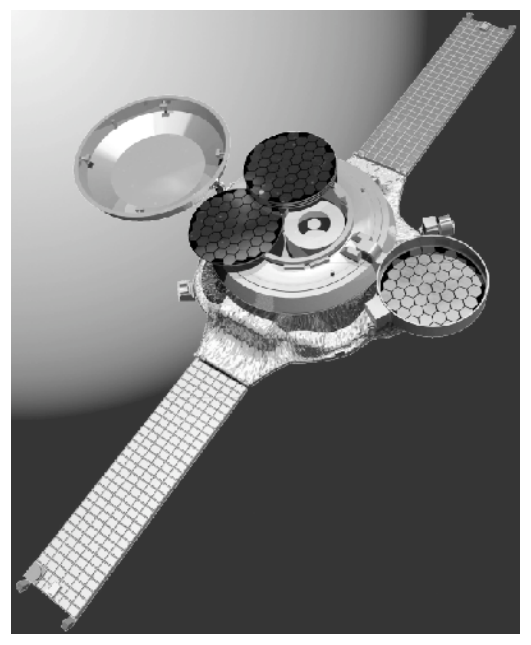

Fig. 1 Genesis spacecraft sampling configuration.

Upon Earth return on September 8, 2004, the entry capsule containing the solar wind samples was released from the main spacecraft, and descended through the Earth's atmosphere (decelerating with the aid of a parachute) for a mid-air recovery using a helicopter over the U.S. Air Force's Utah Test and Training Range (UTTR) in Northwest Utah. Unfortunately, due to a design flaw (the G-switch utilized for drogue deployment was installed upside down), the signal to initiate parachute deployment failed and the capsule subsequently impacted the surface.

This paper provides an overview of the entry, descent, and landing (EDL) trajectory analysis that was performed during the Genesis mission operations phase upon final approach to Earth. In addition, the variation in the predicted landing location and the resulting overall 99 percentile footprint ellipse (obtained from a Monte Carlo analysis) over the final days and hours prior to entry is also presented. This analysis was required in order to substantiate the robustness of the capsule descent to assure that all entry mission requirements were satisfied prior to gaining authorization for capsule separation from the main spacecraft.

\section{Entry, Descent, and Landing Overview}

The Genesis sample return capsule (SRC) is approximately $1.5 \mathrm{~m}$ in diameter. Its forebody is a blunted $60 \mathrm{deg}$ half-angle sphere-cone. The afterbody is a bi-conic backshell with a first cone turning angle of $20 \mathrm{deg}$ and a second cone turning angle of $61.6 \mathrm{deg}$ (Fig. 2). 


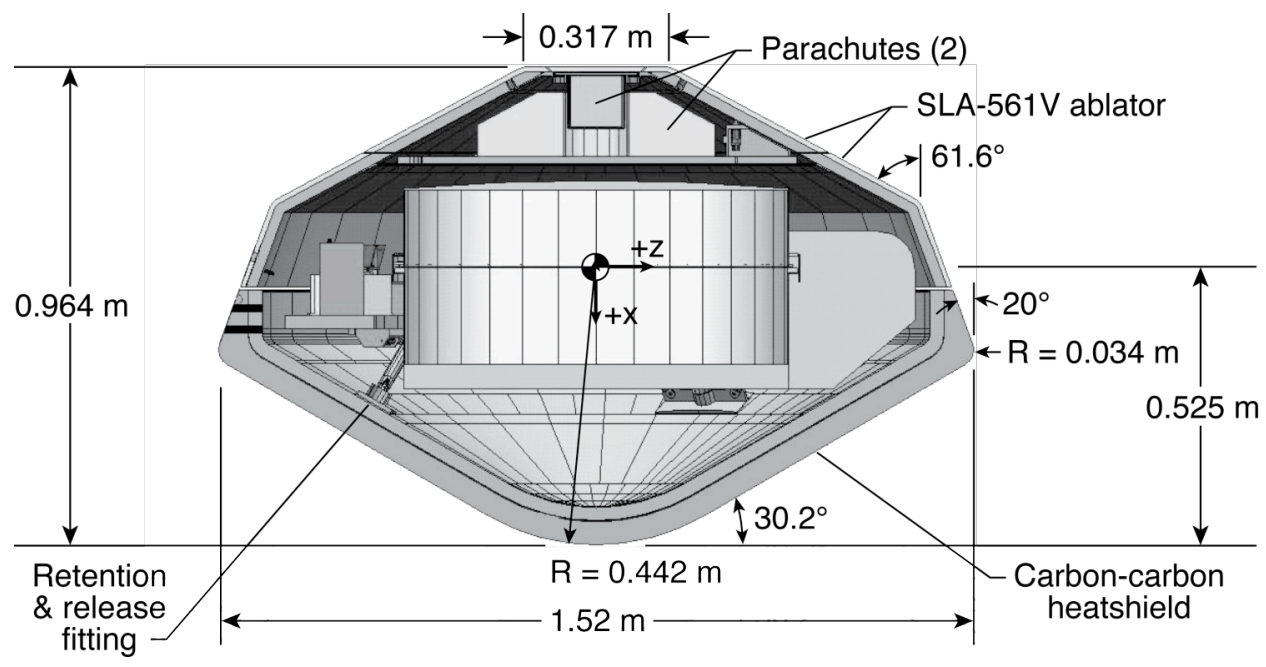

Fig. 2 Genesis SRC configuration.

Figure 3 shows the nominal entry sequence, with the terminal descent phases highlighted. Four hours prior to entry, the $205.6 \mathrm{~kg}$ Genesis SRC was spun-up to $15 \mathrm{rpm}$ and separated from the main spacecraft. The SRC had no active guidance or control systems, so the spin-up was required to maintain its entry attitude (nominal 0 deg angleof-attack) during coast. Throughout the atmospheric entry, the passive SRC relied solely on aerodynamic stability for performing a controlled descent through all aerodynamic flight regimes: free molecular, hypersonic-transitional, hypersonic-continuum, supersonic, transonic, and subsonic. The SRC must possess sufficient aerodynamic stability to overcome the gyroscopic (spin) stiffness in order to minimize any angle-of-attack excursions during the severe heating environment. Additionally, this stability must persist through the transonic and subsonic regimes to maintain a controlled attitude at main parachute deployment. The nominal design inertial entry velocity and inertial flightpath angle for Genesis were $11.04 \mathrm{~km} / \mathrm{s}$ and $-8.0 \mathrm{deg}$, respectively. 


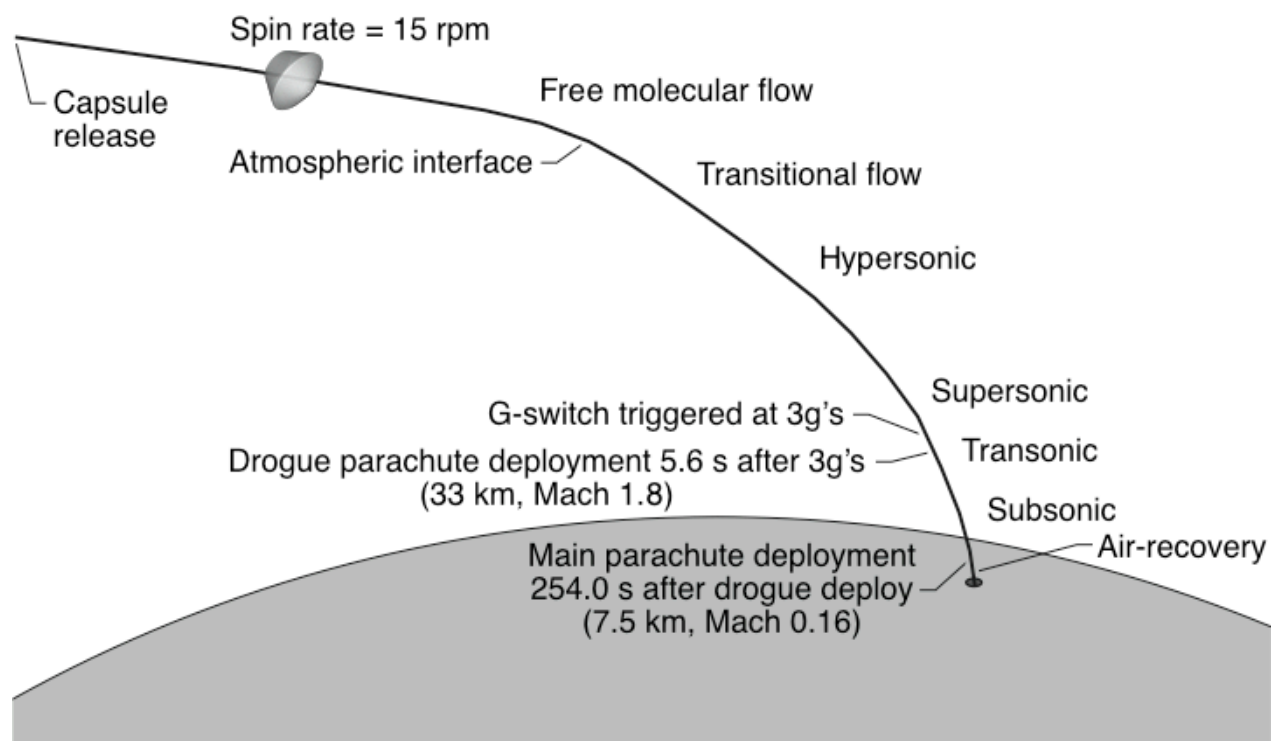

Fig. 3 Nominal Genesis SRC entry sequence.

Reference 2 provides an in-depth description on the development of the entry scenario; specifically, the use of the high spin rate and a supersonic drogue parachute. During descent, the SRC utilizes a G-switch (i.e., gravityswitch) and two timers for deployment of the drogue and main parachutes. The G-switch is triggered after sensing 3 g's (on the decelerating side). At that point, the drogue timer is initiated. After 5.6 seconds, the drogue parachute is deployed (approximately Mach 1.8), and the main timer is initiated. After 254.0 seconds (approximately at $7.5 \mathrm{~km}$ ), the main parachute is deployed. A helicopter air-recovery of the SRC was to occur at an altitude of approximately $2.45 \mathrm{~km}$. This nominal entry sequence was sufficiently robust to accommodate off-nominal conditions during the descent as shown by the Monte Carlo dispersion analyses in Ref. 2.

\section{Earth Return Overview}

The Genesis event timeline for final Earth approach is shown in Figure 4, which highlights the trajectory correction maneuvers (TCM) that were baselined for attaining the proper entry conditions. Reference 1 provides an overview of the entire Earth return strategy showing all of the required TCMs. Prior to TCM-10, which occurred at entry (E) minus 10 days, the Genesis return trajectory was on a path that missed the Earth. Only after TCM-10 was successfully executed did the trajectory of the SRC become targeted at the Earth, with placement of the nominal landing location being in the western portion of UTTR. 


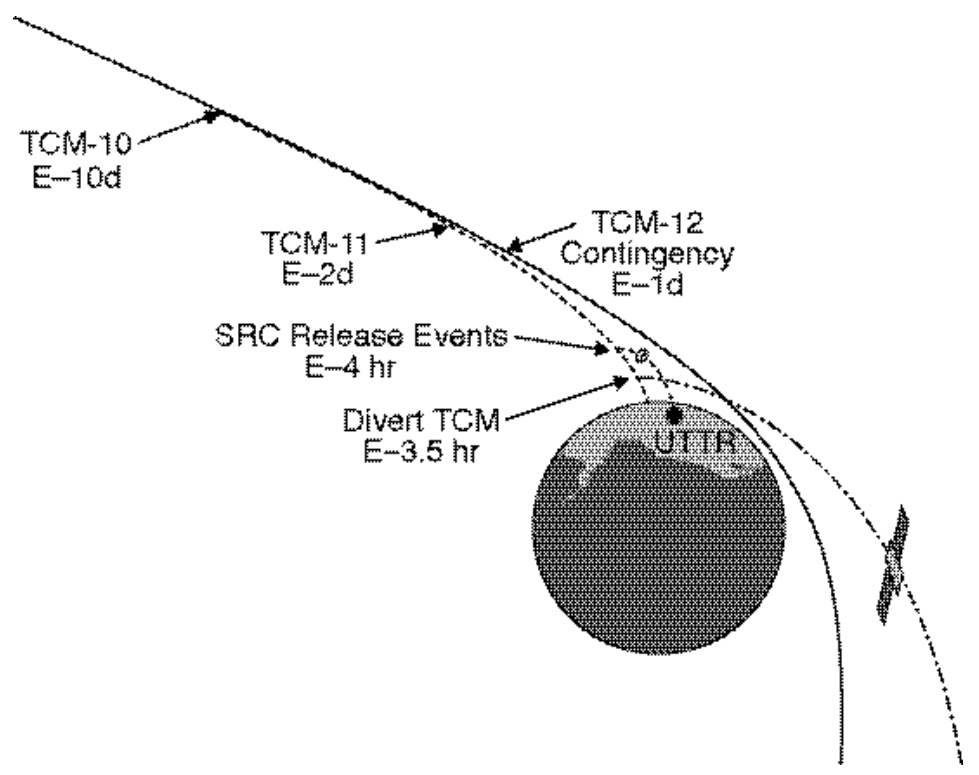

Fig. 4 Genesis final Earth approach event timeline.

Final targeting was accomplished with TCM-11 at E-2 days, which shifted the nominal landing location to the desired center of UTTR. If TCM-11 had not executed or only partially executed, a contingency maneuver TCM-12 would have been implemented at E-1 day to achieve the final desired landing location. At E-4 hours, the SRC was separated from the main spacecraft, thus starting the EDL sequence illustrated in Fig. 3. At E-3.5 hours, a TCM was performed to divert the main spacecraft into an orbit ahead of the Earth. ${ }^{3}$ If TCM-10, 11, and 12 had all been unsuccessful, the capsule/main spacecraft would have flown by the Earth as depicted in Fig. 4. During mission operations, both TCM-10 and TCM-11 executed very successfully, as did the separation and divert maneuvers. As a result, the desired entry conditions were achieved with high accuracy as discussed in Section IV. ${ }^{4}$

\section{Trajectory Simulation}

The Genesis atmospheric entry trajectory is designed to fit within an envelope of derived requirements and physical constraints based upon the capsule hardware design. As such, for a successful landing, all entry requirements must be satisfied. Table 1 lists all the EDL requirements and their specific bounds. Monte Carlo dispersion analyses, described in subsequent sections, were performed during the mission operations phase to assess the satisfaction of these requirements ${ }^{2}$, along with those for public safety as described in detail in Ref. 5. 
Table 1: EDL Requirements and Constraints

\begin{tabular}{lc}
\hline \multicolumn{1}{c}{ Requirement } & Limit \\
\hline Entry Flight-Path Angle Error, deg & $< \pm 0.08$ \\
Entry Velocity, km/s & $<11.07$ \\
Entry Attitude, deg & $<10$ \\
Max Heat Rate, W/cm ${ }^{2}$ & $<510$ \\
Attitude at Max Heat Rate, deg & $<10$ \\
Max Heat Load, KJ/cm ${ }^{2}$ & $<16.6$ \\
Max Deceleration, Earth g & $<40$ \\
Drogue Chute Deployment Attitude, deg & $<30$ \\
Drogue Chute Deployment Mach Number & $>1.1 \&<2.3$ \\
Main Parachute Altitude, km & $>6.7$ \\
Landed Footprint, km & $<84$ \\
\hline \hline
\end{tabular}

\section{A. Monte Carlo Uncertainty Sources}

During the entry, off-nominal conditions may arise that affect the descent profile. These off-nominal conditions can originate from numerous sources: capsule mass property measurement uncertainties; separation attitude and attitude rate uncertainties; limited knowledge of the flight-day atmospheric properties (density and winds); computational uncertainty with the aerodynamics; and uncertainties with parachute deployment. In the analysis, an attempt was made to conservatively quantify and model the degree of uncertainty in each mission parameter. For this entry, 41 potential uncertainties were identified. Table 2 captures these uncertainty sources, respectively, along with their corresponding 3- $\sigma$ variances. Reference 2 provides an in depth description of the analysis methodology developed and utilized during the Genesis capsule design phase and the quantification of the various uncertainty sources. The subsequent sections describe in greater detail a few of the key uncertainty sources that were updated during the mission operations phase prior to the entry as more knowledge was gained. 
Table 2: Monte Carlo Analysis Variables

\begin{tabular}{lcc}
\hline \multicolumn{1}{c}{ Variable } & 3- $\boldsymbol{\sigma}$ Variation & Distribution \\
\hline Entry states & Based on covariance (See Ref. 4) & - \\
Mass, kg & \pm 0.25 & Uniform \\
Radial center-of-gravity offset, mm & \pm 0.71 & Uniform \\
Axial center-of-gravity, mm & \pm 0.71 & Uniform \\
Moments of Inertia (Ixx, Iyy, Izz) & $\pm 5 \%, \pm 5 \%, \pm 5 \%$ & Uniform \\
Cross products (Ixy, Ixz, Iyz), kg-m ${ }^{2}$ & $\pm 0.06, \pm 0.06, \pm 0.75$ & Uniform \\
Entry pitch and yaw attitude, deg & $\pm 3.77, \pm 4.03$ & Gaussian \\
Entry pitch and yaw rates, deg/s & $\pm 3.61, \pm 1.78$ & Gaussian \\
Entry roll rate, deg/s & \pm 6.04 & Gaussian \\
Aerodynamic coefficients & See Ref. 2 & - \\
Drogue Parachute $C_{D}$ & $\pm 10 \%$ & Uniform \\
Main Parachute $C_{D}$ & $\pm 10 \%$ & Uniform \\
G-switch acceleration trigger value & $\pm 10 \%$ & Uniform \\
Drogue parachute timer, $\mathrm{s}$ & \pm 0.05 & Uniform \\
Main parachute timer, $\mathrm{s}$ & \pm 0.05 & Uniform \\
Atmosphere & GRAM- 95 model (See Ref. 6) & - \\
\hline
\end{tabular}

\section{B. Entry Covariance}

The original Genesis strategy for Earth approach was revised to maximize public safety in light of possible anomalies and contingencies, while still preserving the capability to meet the nominal entry requirements. ${ }^{1}$ As a result, a series of maneuvers were performed to set up the approach and entry (see Ref. 1). Initial conditions at entry were obtained from orbit determination solutions performed by the Genesis Navigation Team. Reference 4 gives a description of the navigation process during the return phase and the determination of the final arrival conditions prior to entry. The navigation accuracy obtained for Genesis yielded extremely small state errors upon Earth arrival (well below the 3- $\sigma$ design requirement of \pm 0.08 deg fight path angle error, see Table 1). The final orbit determination solution produced an inertial entry flight-path angle of -8.002 deg (which was the desired nominal value) with a 3 - $\sigma$ error of $\pm 0.0274 \mathrm{deg}$ (a third of the requirement).

\section{Capsule/Cruise-Stage Separation}

Based on the final main spacecraft and capsule mass properties, a statistical separation analysis was performed to predict separation attitude and attitude rate errors. The attitude errors predicted in pitch and yaw were $\pm 3.77 \mathrm{deg}$ and $\pm 4.03 \mathrm{deg}$, respectively. The attitude pitch and yaw rate errors predicted were $\pm 03.61 \mathrm{deg} / \mathrm{s}$ and $\pm 1.78 \mathrm{deg} / \mathrm{s}$, 
respectively, and the roll rate error was $\pm 6.04 \mathrm{deg} / \mathrm{s}$. These variations were used as inputs in the Monte Carlo analysis described in a subsequent section.

\section{Atmosphere Model}

The Earth atmosphere model utilized by Genesis for the entry trajectory design and analysis was the Global Reference Atmospheric Model - 1995 Version (GRAM-95). ${ }^{6}$ This model is an amalgam of three empirically based global data sets of the Earth that can produce an atmosphere profile as a function of altitude for a given date, time, and positional location about the Earth. GRAM-95 produces a representative atmosphere taking into account variations in diurnal, seasonal, and positional information for a given trajectory to produce nominal density, temperature, and pressure profiles and their statistical perturbations along the trajectory flight track. GRAM-95 is not a predictive model, but rather provides a representative atmosphere profile for the given date, time, and positional inputs. A nominal profile was generated using the GRAM-95 model (which is anchored by historical data) for a given date, time, and location. In addition, GRAM-95 also provides statistical perturbations for all the atmosphere parameters (i.e., dispersed profiles).

Figure 5 shows samples of five randomly perturbed density profiles as a percentage of the nominal profile for the Genesis entry date of September 8, 2004 produced by the GRAM-95 model. Also, depicted are the upper and lower $( \pm 3-\sigma)$ boundaries of the possible density variation. In addition, GRAM-95 can also produce nominal wind profiles and their statistical perturbations for the northward, eastward, and vertical wind components. Figures 6 and 7 show five randomly sampled wind profiles (for the Genesis entry date of September 8, 2004) for the northward and eastward wind components, respectively, along with their upper and lower $( \pm 3-\sigma)$ boundaries. In the Monte Carlo

analysis, an atmosphere profile (density and wind components) was randomly generated for each case having the characteristics shown in Figures 5-7. 


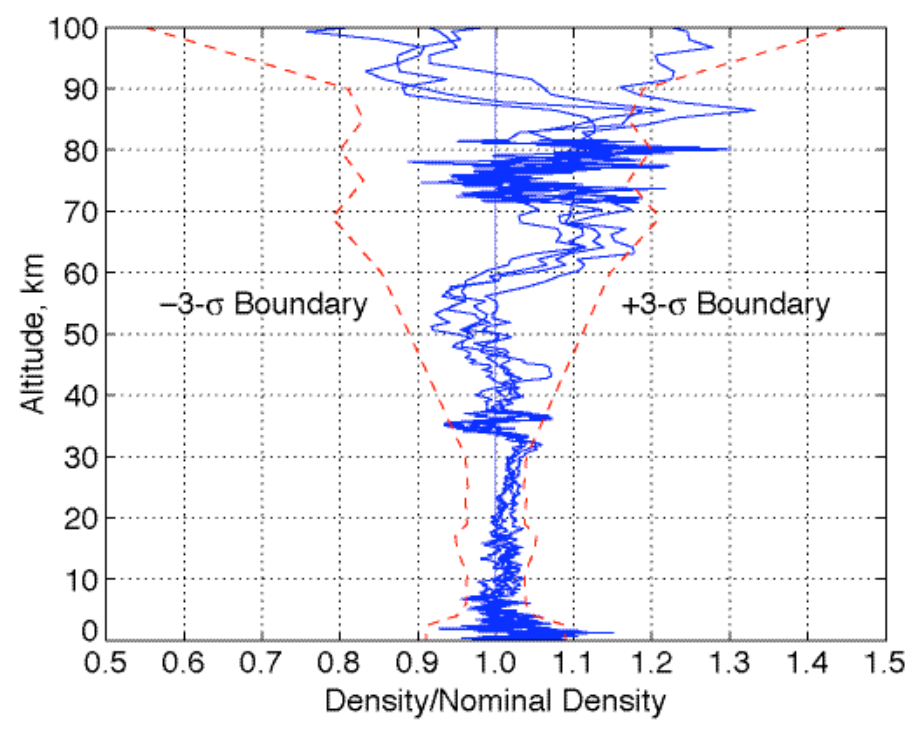

Fig. 5 Density variation from GRAM-95 model.

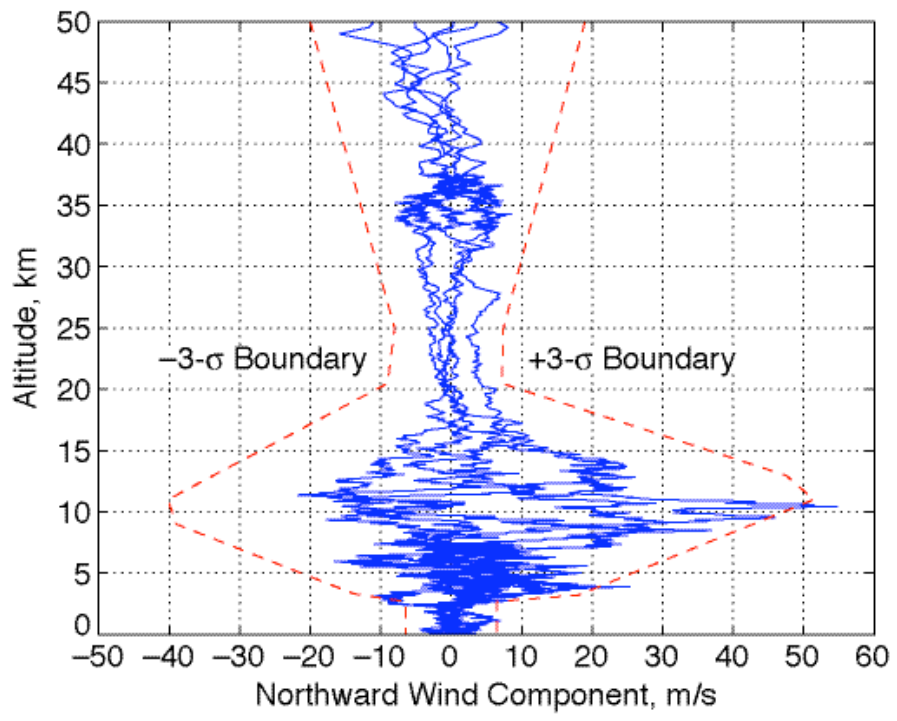

Fig. 6 Northward wind component variation from GRAM-95 model. 


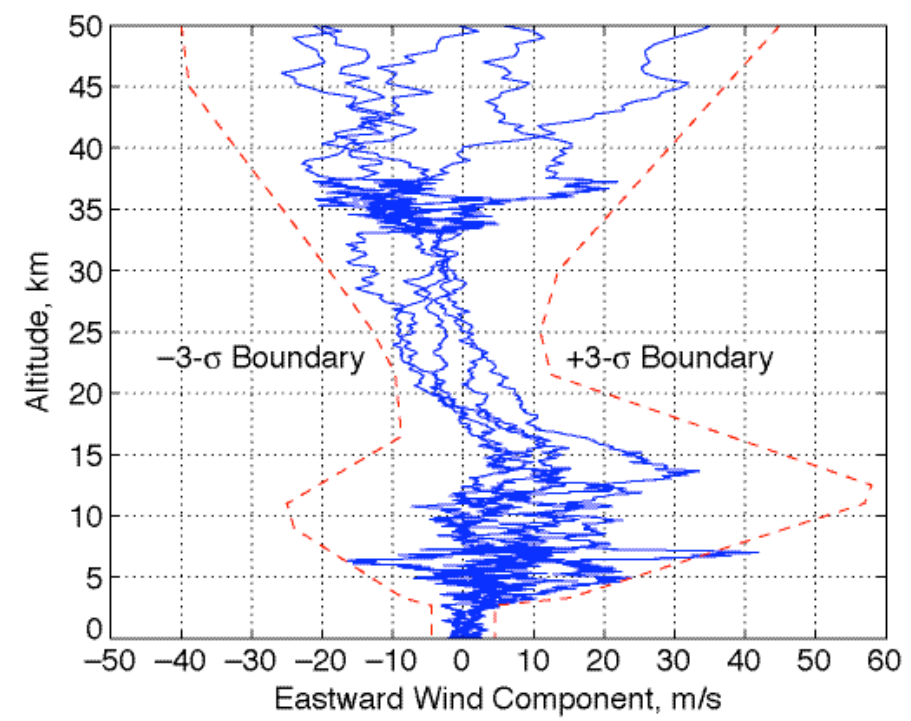

Fig. 7 Eastward wind component variation from GRAM-95 model.

\section{E. Trajectory Analysis}

Two trajectory propagation codes were utilized for the Genesis landing dispersion analyses: the Program to Optimize Simulated Trajectories (POST) program $^{7}$ and the Atmospheric-Entry Powered Landing (AEPL) program. ${ }^{8}$ These codes were employed to obtain independent verification of the predicted nominal landing location and the overall size of the dispersed landing footprint ellipse to ensure public safety by landing within the UTTR boundaries.

The POST trajectory analysis was performed modeling six-degree-of-freedom (6DOF) dynamics, which included all forces and torques on the spacecraft, from atmospheric interface to drogue parachute deployment. During this portion of the entry, the full set of capsule aerodynamics and mass properties were incorporated into the simulation to accurately model the hypersonic descent. From drogue parachute deployment to landing, three-degreeof-freedom (3DOF) dynamics were used, in which only the drag force was modeled and was assumed to act opposite the wind-relative velocity vector. The POST trajectory simulation seamlessly transitions from $6 \mathrm{DOF}$ to 3DOF dynamics within a single continuous simulation.

The version of the AEPL program used for Genesis employed 3DOF analyses throughout. Since the Genesis entry was unguided and ballistic, the 3DOF results from AEPL agreed well with the POST 6DOF/3DOF simulation. Between the two simulations, the POST results were baselined as primary for the mission. In general, there was very good agreement between the two simulations. 


\section{F. Entry Analysis Timeline}

During Earth approach operations, entry analyses (described in the next section) had to be completed on a specific timeline to allow decisions to be made regarding the Genesis landing. The sequence of events prior to entry is listed in Table 3 (all times are relative to entry interface). TCM-11 at Entry -48 hours is listed as the first event, because it is the maneuver that points Genesis to the desired target in the center of UTTR. Two "GO/NO-GO" meetings (occurring at Entry -35 hours and Entry -15 hours) were held to review whether the EDL (see Table 1) and public safety (see Ref. 5) requirements were satisfied. The GO/NO-GO meetings were where the decisions were made to authorize Earth return. The GO/NO-GO meetings 1 and 2 were the two most important times when the entry analyses results had to be available to assure that all EDL and public safety requirements were satisfied, since once the SRC was released from the main spacecraft at Entry -4 hours, there was nothing that can be done to modify the trajectory of the capsule. The entry results at both of these GO/NO-GO meetings showed compliance of all requirements and lead to decisions to authorize the entry. At Entry -10 hours, the entry analyses was repeated with the final orbit determination solution for the entry state vector that was available prior to the SRC Release GO/NOGO Meeting at Entry -8 hours. This meeting was the final opportunity to abort the entry if there were indications of any issues. The results were very similar to those at the GO/NO-GO Meeting 2, showing compliance of all EDL and public safety requirements. Consequently, the entry sequence was allowed to continue for SRC release from the main spacecraft and subsequent entry. The predicted landing location from the SRC Release GO/NO-GO Meeting results at Entry -8 hours was used to judiciously position the helicopter in UTTR to minimize the distance between it and the descending capsule for the air-recovery. Unfortunately, due to a design flaw (the G-switch utilized for drogue deployment was installed upside down), the signal to initiate parachute deployment failed and the capsule subsequently impacted the surface. Consequently, the air-recovery was not attempted.

Table 3: Timeline for Entry

\begin{tabular}{cc}
\hline \hline Time & Event \\
\hline Entry -48 hours & Execution of TCM-11 \\
Entry -35 hours & GO/NO-GO Meeting \#1 \\
Entry -24 hours & Execution of Contingency TCM-12 \\
Entry -15 hours & GO/NO-GO Meeting \#2 \\
Entry -8 hours & SRC Release GO/NO-GO Meeting \\
Entry -4 hours & SRC Release or Divert \\
Entry +0 & Entry Interface \\
\hline \hline
\end{tabular}


Leading up to the GO/NO-GO Meeting 1 and 2 decisions, the timeline was compressed. The entry, descent, and landing analyses (Monte Carlo and public safety) had to be performed within $90 \mathrm{~min}$, and compared to the requirements. Great amount of effort was spent practicing and streamlining this operation prior to these meetings. The 90 min were split with 45 min allocated for the Monte Carlo dispersion analysis and 30 min for the public safety analysis (described in Ref. 5), which required the Monte Carlo analysis results as input. The remaining 15 min were allocated for presentation material preparation. In general, 90 min were sufficient for completing the Monte Carlo dispersion and public safety analyses. Since the entry inertial fight-path angle attained was the desired target of -8.0 deg with a 3- $\sigma$ error of only \pm 0.0274 deg (a third of the requirement), all the EDL and public safety metrics were well within their requirements and easily satisfied.

In general, a violation of anyone of the EDL or public safety requirements at the GO/NO-GO meetings would lead to a NO-GO decision for landing; however, in some cases, discretion was given to the project manager as to whether the Genesis capsule would be authorized to separate from the main spacecraft (at Entry -9 hours). However, all events performed nominally, and there were no EDL or public safety requirement violations. The contingency maneuver TCM-12 at Entry -24 hours was not required. Operationally, all the requirements were monitored weeks prior to the final approach and not just at these GO/NO-GO meeting times. Overall, the Earth approach operation procedures worked well and there were no issues (logistically or performance based) that arose.

\section{G. Monte Carlo Entry Trajectory Dispersion Analysis}

A Monte Carlo dispersion analysis was utilized to statistically assess the robustness of the entry to off-nominal conditions to assure that all EDL requirements and constraints are satisfied (see Table 1). All the input variables listed in Table 2 were randomly varied in the Monte Carlo dispersion analysis, along with their respective variance and distribution type. The analysis included uncertainties in the initial state vector, capsule mass properties (mass, center-of-gravity, inertia), initial attitude and attitude rates, aerodynamic coefficients, atmospheric density and winds, parachute drag, g-switch trigger value, and parachute deployment timers.

For the dispersion analysis, 3000 random cases were run for all the navigation orbit determination (OD) solutions that were computed ${ }^{4}$ at the various event times during the mission operations phase. This analysis was performed to determine the appropriate magnitude and direction of TCM-10 and TCM-11 for proper targeting to UTTR. In addition, this analysis was used to assess the OD solution stability and to understand the movement of the nominal landing location and the variation in the 99 percentile footprint size within UTTR. This understanding was 
crucial in order to gain authorization for capsule separation and the subsequent entry. The size of the 99 percentile footprint ellipse obtained from the Monte Carlo dispersion analysis was used in a public safety analysis to certify that the risks of the Genesis capsule entry were accepatable. ${ }^{5}$

The Monte Carlo entry trajectory dispersion analysis was performed on all the post-TCM10 OD solutions (OD139 through OD144). Figure 8 shows the corresponding results at landing. For clarity, only the results for three OD solutions are shown (OD139, OD141, and OD144), where their nominal landing locations (center points) and the 99 percentile footprint ellipses at UTTR are depicted. As previously stated, TCM-10 targets the nominal landing location towards the western portion of UTTR (approximately $55 \mathrm{~km}$ from the target). The target location selected for Genesis was near the center of UTTR having the coordinates 246.4667 deg East Longitude and 40.2 deg North Latitude.

Over the course of post-TCM10 OD solutions, the nominal landing location was observed to drift as expected. The movement was first towards the Southeast between OD139 through OD141, before reversing back towards the Northwest between OD141 through OD144. The later OD solutions began to stabilize as more tracking data was available due to the increased observation time. The landing locations for OD142 through OD144 are hard to differentiate, as they lie nearly on top of each other. The added benefit of stable OD solutions (arising from the increased observation time) was that a refinement in the footprint size was obtained. For the post-TCM10 OD solutions, the 99 percentile footprint ellipses decreased in size. The footprint size decreased from $69.7 \mathrm{~km}$ by 27.7 $\mathrm{km}$ for OD139 to $46.5 \mathrm{~km}$ by $27.1 \mathrm{~km}$ for OD141 to $37.1 \mathrm{~km}$ by $26.7 \mathrm{~km}$ for OD144.

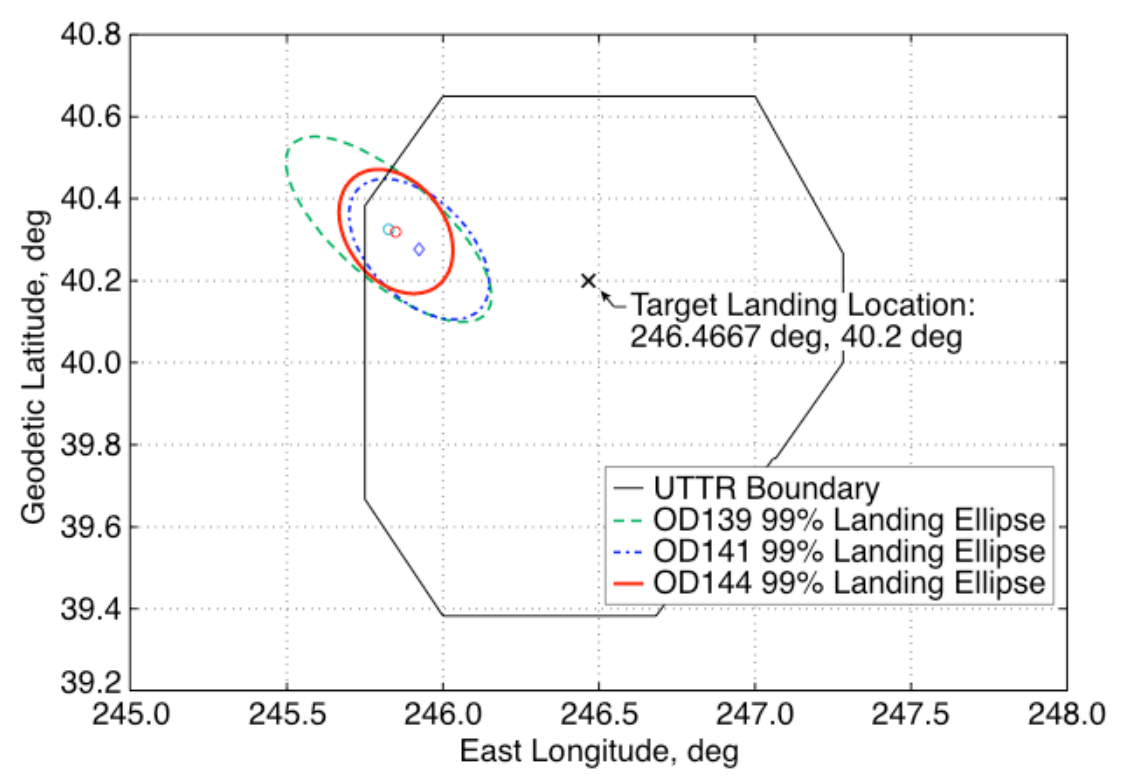


Fig. 8 Landing location for post-TCM10 OD solutions.

Similarly, Monte Carlo analyses were performed for all the post-TCM11 OD solutions (OD150 through OD154). Figure 9 depicts the landing locations for a few of these OD solutions (OD150, OD152, and OD154). As seen, TCM11 moved the landing location to the center from the western portion of UTTR. All the OD solutions postTCM11 were extremely stable and produced nearly identical landing locations as observed in Fig. 9, where the 99 percentile landing ellipses lie nearly on top of each other. The results for OD154 showed that all the EDL and public safety requirements and constraints were well within their limits, and that the final predicted nominal landing location was very close to the desired target (only $1.65 \mathrm{~km}$ away). The final overall 99 percentile landing ellipse was calculated to be $41.9 \mathrm{~km}$ by $21.1 \mathrm{~km}$ having an azimuth orientation angle of $137.2 \mathrm{deg}$ (measured clockwise positive from north). Based on these OD154 results (which was the last OD solution available prior to entry), the authorization for capsule separation and subsequent entry was granted. Unfortunately, due to the design flaw (the Gswitch utilized for drogue deployment was installed upside down), the signal to initiate drogue parachute deployment failed, and the capsule subsequently tumbled and impacted the surface. The final impact point was 8.3 $\mathrm{km}$ south of the desired target as shown in Fig. 10, which was well within the final OD154 pre-entry predicted 99 percentile landing ellipse. Post-flight reconstruction indicates that the Genesis entry was very close to the pre-entry predictions. Reference 9 provides a detailed overview of flight reconstruction and comparison to pre-entry predictions.

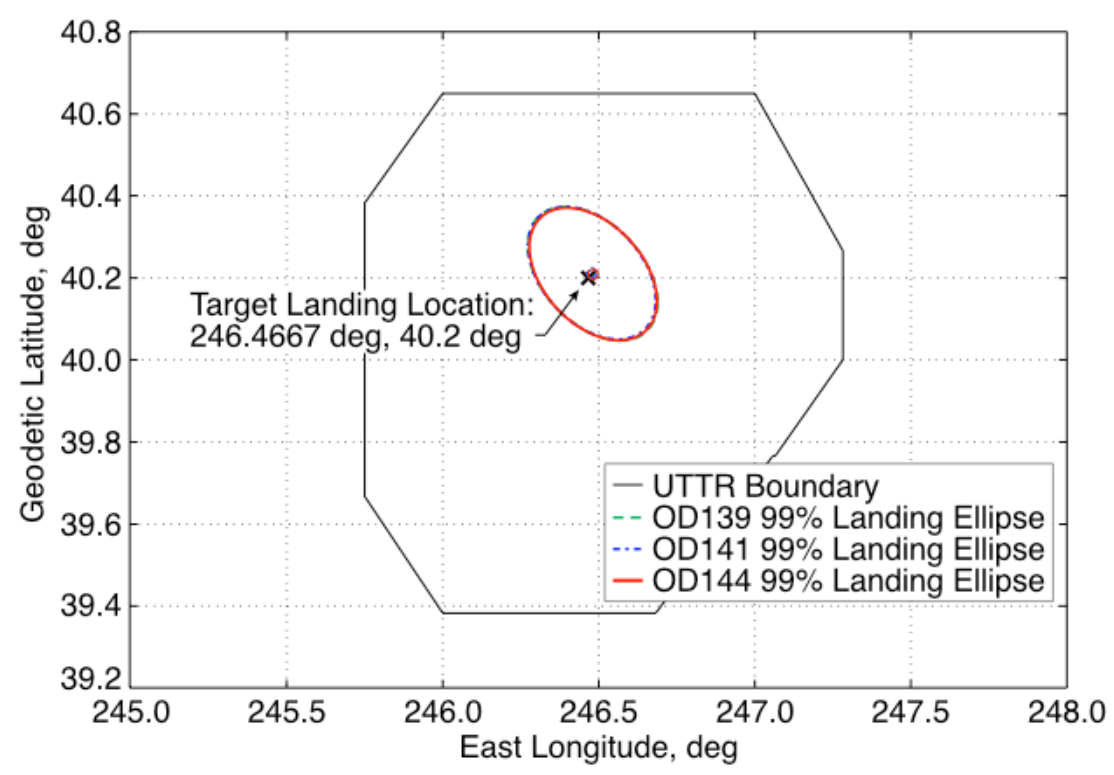

Fig. 9 Landing location for post-TCM11 OD solutions. 


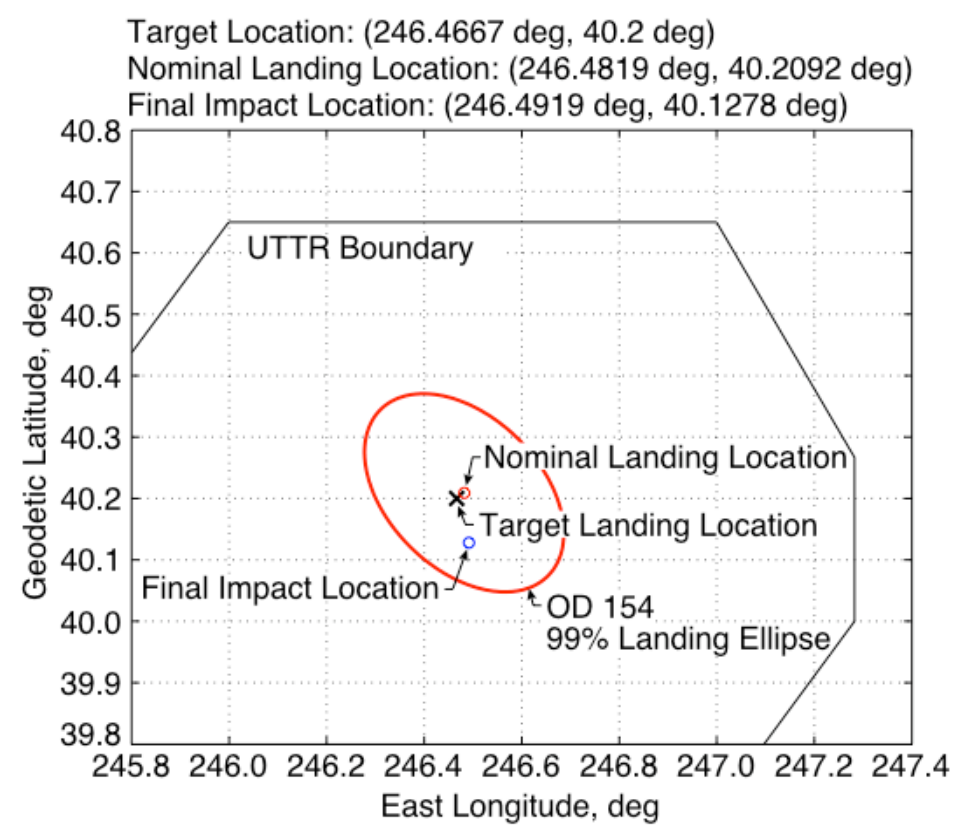

Fig. 10 Landing location for Post-TCM11 OD solutions.

\section{Conclusions}

On September 8, 2004, the Genesis entry capsule containing the solar wind samples was released from the main spacecraft, and descended through the Earth's atmosphere. The final orbit determination solution produced an inertial entry flight-path angle of $-8.002 \mathrm{deg}$ (which was the desired nominal value) with a $3-\sigma$ error of $\pm 0.0274 \mathrm{deg}$ (a third of the requirement). The entry, descent, and landing trajectory analyses that were performed during the mission operations phase upon final approach to Earth accurately delivered the entry capsule to the desired landing site. The capsule landed $8.3 \mathrm{~km}$ from the desired target, and was well within the allowable landing area at the Utah Test and Training Range. The entry, descent, and landing analyses had to be performed within a 90 min timeframe to allow sufficient time for performing all the Earth approach procedures. All events performed nominally, and there were no entry requirement violations. Overall, the Earth approach operation procedures worked well and there were no issues (logistically or performance based) that arose. As a result, the process of targeting a capsule from deep space and accurately landing it on Earth was successfully demonstrated.

\section{Acknowledgments}

The AEPL trajectory simulation portion of this research was carried out at the Jet Propulsion Laboratory, California Institute of Technology, under a contract with the National Aeronautics and Space Administration. The 
authors would like to acknowledge the contributions of the other members of the Navigation Team for the exceptional effort in achieving such accurate targeting for the Genesis entry.

\section{References}

${ }^{1}$ Williams, K. E., Lewis, G. D., Helfrich, C. E., Wilson, R. S., and Potts, C. L., "Genesis Earth Return: Refined Strategies and Flight Experience," AAS 05-116, January 2005.

${ }^{2}$ Desai, P. N., Mitcheltree, R. A., and Cheatwood, F. M., "Entry Dispersion Analysis for the Genesis Sample Return Capsule," Journal of Spacecraft and Rockets, Vol. 38, No. 3, 2001, pp. 345-350.

${ }^{3}$ Wilson, R. S., “Genesis Extended Mission Trajectory Design,” AAS 05-226, January 2005.

${ }^{4}$ Han, D., Lewis, G. D., Wawrzyniak, G. G., Graat, E. J., Craig, D. E., Baird, D. T., and Bhaskaran, S., "Genesis Orbit Determination for Earth Return and Atmospheric Entry,” AAS 05-117, January 2005.

${ }^{5}$ Wawrzyniak, G., and Wahl, T., "Human Safety Analysis for the Genesis Sample Return Mission,” AAS 05-223, January 2005.

${ }^{6}$ Justus, C. G., Jeffries III, W. R., Yung, S. P., and Johnson, D. L., “The NASA/MSFC Global Reference Atmospheric Model - 1995 Version (GRAM-95),”NASA TM-4715, Aug. 1995.

${ }^{7}$ Brauer, G. L., Cornick, D. E., and Stevenson, R., "Capabilities and Applications of the Program to Optimize Simulated Trajectories (POST),” NASA CR-2770, Feb. 1977.

${ }^{8}$ Klumpp, A. R., Atmospheric-Entry, Powered Landing Simulator V4.10C User's Guide, April 23, 2003 (JPL Internal Document).

${ }^{9}$ Desai, P. N., Qualls, G. D., and Schoenenberger, M., "Reconstruction of the Genesis Entry," companion manuscript accepted for publication (log A12603) in the Journal of Spacecrafts and Rockets. 